Impression of Change (CGIC), and safety in younger ( $<55$ years) and older ( $\geq 55$ years) patients.

Results. This analysis included 119 younger and 218 older patients enrolled in the OLE. Data presented at Week 145 (mean \pm SE): total deutetrabenazine dose was $39.4 \pm 1.39 \mathrm{mg} /$ day and $39.5 \pm 1.04 \mathrm{mg} /$ day in younger and older patients, respectively. Changes from baseline in AIMS score were $-6.7 \pm 0.62$ and $-6.5 \pm 0.47$, respectively (percent changes of $-61.4 \% \pm 4.10 \%$ and $-54.6 \% \pm 3.01 \%$ ). The majority of younger and older patients achieved treatment success per CGIC (67\% and 76\%) and PGIC (64\% and 63\%) and achieved $\geq 50 \%$ AIMS response ( $76 \%$ and $62 \%$ ). Deutetrabenazine was generally well tolerated in both groups. Exposure-adjusted incidence rates (incidence/patient-years) were $<0.01$ and 0.02 for akathisia, 0.07 (both) for somnolence and sedation, 0.04 and 0.11 for parkinson-like events, and 0.06 and 0.09 for depression in younger and older patients, respectively.

Conclusions. Deutetrabenazine treatment was associated with sustained improvements in AIMS score and was well tolerated in both younger and older TD patients.

Funding. Teva Pharmaceutical Industries Ltd., Petach Tikva, Israel

\section{Analysis of Treatment Goals for Patients with Schizophrenia: A US Survey of Psychiatrists, Patients with SCZ and Caregivers}

\author{
Maxine Chen, $\mathrm{PhD}^{3}$, Jason Shepherd, $\mathrm{BA}^{2}$, \\ Hollie Bailey, $\mathrm{BSc}^{2}$, Jack Wright, $\mathrm{MSc}^{2}$ and \\ Heather M. Fitzgerald, PharmD ${ }^{1}$
}

${ }^{1}$ Lundbeck LLC, Deerfield, IL, USA, ${ }^{2}$ Adelphi Real World, Bollington, Cheshire, United Kingdom, and ${ }^{3}$ Otsuka Pharmaceutical Development \& Commercialization, Inc., Princeton, NJ, USA

Presenting Author: Maxine Chen

\begin{abstract}
Objectives. Objectives for this survey are to determine similarities or differences in treatment goals reported by psychiatrists, patients with schizophrenia (SCZ) and caregivers in the US, as well as whether goals differed by patients currently on an oral antipsychotic (OAP) or long-acting injectable (LAI), and whether goals differed by age.

Methods. This was a real-world, cross-sectional survey of US psychiatrists, patients $=18$ years old diagnosed with SCZ, and caregivers. Data was collected using the Disease Specific Programme (DSP) methodology. Psychiatrists $(n=120)$ completed detailed record forms for next 8 outpatients and 2 inpatients matching inclusion criteria. The same patients and their caregivers, if present, were invited by their psychiatrist to voluntarily complete a separate survey.

Results. Responses on treatment goals were collected from psychiatrists for all patients included in the analysis $(n=1161)$, patients $(n=542)$ and caregivers $(n=130)$. Among 3 top goals,
\end{abstract}

psychiatrists, patients and caregivers concurred that "decrease in disease symptoms" is most important $(63 \%, 64 \%, 68 \%$ respectively). For psychiatrists and caregivers, second was "decrease in hospitalization for relapse" ( $41 \%$, and $38 \%$ respectively), whereas for patients, it was "thinking clearly" (47\%). Of the 3 least important goals, psychiatrists, patients and caregivers agreed with "sexual problems" (59\%, 43\%, 44\%, respectively) and "weight gain" (38\%, 44\%, 38\%, respectively).

When asked which goals were met by current medication, patients responded "decrease in disease symptoms" (68\%) and "thinking clearly" (39\%). However, caregivers responded "thinking clearly" (30\%) was not met by current medication. Caregivers most important goals, "decrease in disease symptom" (70\%) and "decrease in hospitalization for relapse" (41\%), were met. Additional analyses of patients on OAPs and LAIs, did not show differences in goals. However, "decrease in disease symptoms" was numerically more important for patients on LAIs vs OAPs according to psychiatrists ( $68 \%$ vs $62 \%$ ) and caregivers (77\% vs $70 \%$ respectively). Caregivers responded "decrease in hospitalization for relapse" was met for $63 \%$ patients currently on an LAI and 35\% OAP. No major differences in treatment goals were observed by patient age ( $18-35$ vs $36-65$ vs $>65$ years).

Discussion. There is consensus among US psychiatrists, patients and caregivers on the most important treatment goal "decrease in disease symptoms", regardless of patients' current medication or age. For patients, "thinking more clearly" was second, compared with "decrease in hospitalization due to relapse", for psychiatrists and caregivers. All agreed that least important treatment goals, related to AEs, were "weight gain" and "sexual problems". More caregivers agreed "decrease in hospitalization for relapse" was met by patients on LAIs vs OAPs. These findings may help with discussions between psychiatrists, patients and caregivers.

Funding. Lundbeck LLC and Otsuka Pharmaceutical Development \& Commercialization, Inc.

\section{Safety and Tolerability of Aripiprazole Lauroxil 2-Month Formulation With 1-Day Initiation for Treatment of Schizophrenia in the ALPINE Study}

Ilda Bidollari, MD, MBA, Alexa Vasios and

Amy Claxton, PhD

Alkermes, Inc., Waltham, MA, USA

Presenting Author: Ilda Bidollari
Abstract
Objective. Evaluate safety and tolerability of an aripiprazole lauroxil (AL) 2-month regimen using 1-day initiation in patients hospitalized for acute exacerbation of schizophrenia and transi- tioned to outpatient care. 
Methods. In the 25-week, double-blind ALPINE study, adults hospitalized for an acute exacerbation of schizophrenia were randomized to AL (AL NanoCrystal Dispersion + oral aripiprazole $30 \mathrm{mg}$ day 1; AL $1064 \mathrm{mg}$ day 8 and q8wk) or the active control paliperidone palmitate (PP $234 \mathrm{mg}$ day 1; PP $156 \mathrm{mg}$ day 8 and $\mathrm{q} 4 \mathrm{wk}$ ), discharged after 2 weeks if clinically stable, and followed through the end of the study. Adverse events, including adverse events of special interest (AESIs; extrapyramidal symptoms [identified by non-mutually exclusive standardized MedDRA queries], sedation, hypotension, injection site reactions [ISRs], suicidal ideation and behavior) were monitored throughout the study.

Results. In total, 200 patients were randomized ( $\mathrm{AL}, \mathrm{n}=99$; $\mathrm{PP}$, $\mathrm{n}=101$ ); 99 patients (AL, $\mathrm{n}=56 ; \mathrm{PP}, \mathrm{n}=43$ ) completed the study. Rates of AESIs in AL-treated patients were akathisia, $10 \%$; Parkinson-like events, 2\%; dyskinesia, 3\%; dystonia, 9\%; sedation, $7 \%$; hypotension, $6 \%$; ISRs, $18 \%$ (including placebo); and suicidal ideation and behavior, $2 \%$. In PP-treated patients, AESI rates were akathisia, $12 \%$; Parkinson-like events, $4 \%$; dyskinesia, $5 \%$; dystonia, 11\%; sedation, 7\%; hypotension, $4 \%$; ISRs, $27 \%$ (including placebo); and suicidal ideation and behavior, $3 \%$.

Conclusion(s). No unexpected safety and tolerability findings were identified in patients treated with $\mathrm{AL}$ or PP who were hospitalized for acute schizophrenia exacerbation and transitioned to outpatient care in ALPINE. AESI profiles were consistent with each treatment's respective known safety profile.

Funding. Alkermes, Inc.

Disclosures. All authors are employees of Alkermes, Inc., and may own stock/options in the company.

\section{Minimal Clinically Important Difference in AIMS Score Based on CGIC and PGIC in Patients With Tardive Dyskinesia Treated With Deutetrabenazine}

\author{
Hadas Barkay, $\mathrm{PhD}^{1}$, Robert A. Hauser, MD, MBA ${ }^{2}$, \\ Amanda Wilhelm, $\mathrm{PhD}^{3}$, Maria Wieman, $\mathrm{MPH}^{3}$, \\ Mark Forrest Gordon, $\mathrm{MD}^{3}$ and \\ Juha-Matti Savola, MD, $\mathrm{PhD}^{4}$
}

\footnotetext{
${ }^{1}$ Teva Pharmaceutical Industries Ltd., Netanya, Israel, ${ }^{2}$ University of South Florida, Parkinson's Disease and Movement Disorders Center, Tampa, FL, USA, ${ }^{3}$ Teva Pharmaceutical Industries Ltd., West Chester, PA, USA, and ${ }^{4}$ Teva Pharmaceutical Industries Ltd., Basel, Switzerland
}

Presenting Author: Hadas Barkay

\begin{abstract}
Background. Deutetrabenazine is FDA approved for tardive dyskinesia (TD) based on two 12-week, placebo-controlled studies evaluating safety and efficacy in patients with baseline Abnormal Involuntary Movement Scale (AIMS) score $\geq 6$. Deutetrabenazine reduced overall AIMS scores compared with placebo in ARM-TD
\end{abstract}

( -3.0 vs $-1.6, \mathrm{P}=0.019)$ and AIM-TD $(24 \mathrm{mg} / \mathrm{day},-3.2$ vs -1.4 , $\mathrm{P}=0.003 ; 36 \mathrm{mg} /$ day, $-3.3 \mathrm{vs}-1.4, \mathrm{P}=0.001)$. This analysis assessed Minimal Clinically Important Difference (MCID) in AIMS score in patients with TD treated with deutetrabenazine.

Methods. MCID is the smallest change from baseline in AIMS score that is meaningful for patients. MCID analyses were performed based on Patient Global Impression of Change (PGIC) and Clinical Global Impression of Change (CGIC) as anchors described by Hauser et al., where MCID is the difference between patients treated with deutetrabenazine who were minimally improved and patients treated with placebo who were unchanged. Additional MCID definitions were explored: difference between patients who demonstrated treatment improvement versus those who did not (Method 2); difference between patients who demonstrated treatment success versus those who did not (Method 3). Results. 295 patients were analyzed. Based on PGIC, the suggested MCID was -2.8 . Results were similar for Method 2 (75\% of patients had treatment improvement; MCID $=-2.8$ ) and Method 3 (38\% of patients had treatment success; MCID $=-2.6)$. Based on CGIC, the suggested MCID was -2.6. Results were similar for Method 2 (76\% of patients had treatment improvement; MCID = -2.8) and Method 3 ( $41 \%$ of patients had treatment success; MCID $=-3.0$ ). Therefore, the suggested MCID for deutetrabenazine is -3 .

Conclusions. The MCID for change in AIMS score based on PGIC and CGIC for deutetrabenazine was -3 regardless of the analytical method. Findings suggest an AIMS score reduction of $\sim 3$ is associated with clinically meaningful improvement in TD symptoms. Funding. Teva Pharmaceutical Industries Ltd., Petach Tikva, Israel

\section{Effect of Deutetrabenazine on Metabolic Parameters in the Treatment of Tardive Dyskinesia}

\author{
Joohi Jimenez-Shahed, MD ${ }^{1}$, Hadas Barkay, $\mathrm{PhD}^{2}$, \\ Karen E. Anderson, $\mathrm{MD}^{3}$, Hubert H. Fernandez, $\mathrm{MD}^{4}$, \\ Stewart A. Factor, $\mathrm{DO}^{5}$, Robert A. Hauser, MD, MBA ${ }^{6}$, \\ Maria Wieman, $\mathrm{MPH}^{7}$, Mark Forrest Gordon, $\mathrm{MD}^{7}$ and \\ Juha-Matti Savola, MD, $\mathrm{PhD}^{8}$
}

\footnotetext{
${ }^{1}$ Icahn School of Medicine at Mount Sinai, New York, NY, USA, ${ }^{2}$ Teva Pharmaceutical Industries Ltd., Netanya, Israel, ${ }^{3}$ Georgetown University, Washington, DC, USA, ${ }^{4}$ Cleveland Clinic, Cleveland, OH, USA, ${ }^{5}$ Emory University, Atlanta, GA, USA, ${ }^{6}$ University of South Florida, Parkinson's Disease and Movement Disorders Center, Tampa, FL, USA, ${ }^{7}$ Teva Pharmaceuticals, West Chester, PA, USA, and ${ }^{8}$ Teva Pharmaceuticals, Basel, Switzerland
}

Presenting Author: Joohi Jimenez-Shahed

\section{Abstract}

Background. Deutetrabenazine, a novel vesicular monoamine transporter 2 (VMAT2) inhibitor, is approved by the FDA for treatment of tardive dyskinesia (TD) in adults. Dopaminereceptor antagonists (DRAs) are associated with worsening of metabolic parameters, including weight gain, hyperlipidemia, and elevated blood glucose. This post hoc analysis assessed the short- 